\title{
Operational experience and performance of the present ALICE ITS
}

\author{
Serhiy Senyukov* for ALICE collaboration \\ Bogolyubov Institute for Theoretical Physics, CERN \\ E-mail: serhiv.senyukovdcern.ch
}

ALICE (A Large Ion Collider Experiment) is one of four major experiments at the CERN LHC. ALICE studies strongly interacting matter under extreme conditions created in heavy ion collisions. The Inner Tracking System (ITS) is an essential part of the ALICE detector. It is used for tracking, reconstruction of primary and secondary vertices and particle identification. ITS is composed of six cylindrical layers of silicon detectors. Three different techologies are used: hybrid pixel, drift and strip detectors. The ITS was fully commisioned in 2009 at the start of LHC Run 1. The detectors showed good performance during this period contributing to several important measurements. During the LHC Long Shutdown 1 (LS1) the ITS underwent general consolidation and is now ready for the next LHC run.

The 23rd International Workshop on Vertex Detectors,

15-19 September 2014

Macha Lake, The Czech Republic

\footnotetext{
* Speaker.
} 


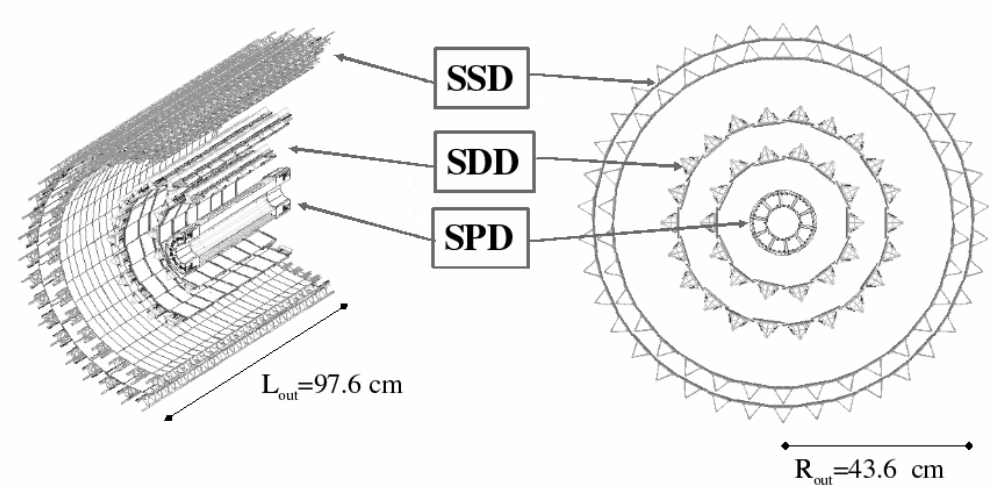

Figure 1: Schematic view of the ITS.

\section{Introduction}

ALICE[四] (A Large Ion Collider Experiment) is one of four major LHC experiments at CERN. Its main goal is to study strongly interacting matter under extreme conditions of temperature and energy density created in ultra-relativistic $\mathrm{Pb}-\mathrm{Pb}$ collisions. The ALICE experimental program also includes pp and $\mathrm{p}-\mathrm{Pb}$ collisions that are used to collect reference data.

The ALICE apparatus is composed of a central barrel $(|\eta|<0.9)$ and a forward muon spectrometer $(2.5<\eta<4.0)$. Detectors of the central barrel are embedded in the solenoidal magnetic field of $B \leq 0.5 \mathrm{~T}$. Among them it is the Inner Tracking System (ITS) that is located closest to the interaction point. The ITS fulfils several tasks in ALICE. First of all, it is used to determine the primary and secondary vertices of the collision event. Then, the ITS complements the track reconstruction done in the Time Projection Chamber (TPC). In combination with the TPC, Transition Radiation Detector (TRD) and Time of Flight (TOF) detector the ITS provides particle identification (PID). Additionally, the two innermost pixel layers of the ITS generate a fast Level-0 trigger with latency below $800 \mathrm{~ns}$.

\section{ITS design overview}

Schematic view of the ITS is shown in Fig. W. The system is composed of six cylindrical layers located at the radii $3.9,7.6,15,23.9,38$ and $43 \mathrm{~cm}$. Longitudinally ITS layers cover the rapidity range of $|\eta|<0.9$ for all vertices located in the range of $\pm 5.3 \mathrm{~cm}$ with respect to the centre of the

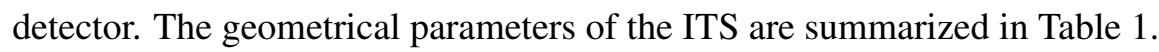

From the point of view of the detector technologies the ITS is divided into three independent sub-detectors: the Silicon Pixel Detector (SPD), the Silicon Drift Detector (SDD) and the Silicon Strip Detector (SSD). The technologies were chosen according to the particle densities at different radii to keep the occupancy at the few percent level at all the layers for the most central $\mathrm{Pb}-\mathrm{Pb}$ collisions.

Silicon Pixel Detector (SPD) The SPD equips the two innermost layers of the ITS and is composed of 240 hybrid pixel modules. Each module consists of one $200 \mu \mathrm{m}$ thick sensor and five $150 \mu \mathrm{m}$ thick readout chips connected via bump-bonding. The sensor is the matrix of $256 \times 160$ 


\begin{tabular}{cccccc}
\hline Layer & Sub-detector & $r[\mathrm{~cm}]$ & $\pm z[\mathrm{~cm}]$ & Nb. of modules & Material budget $\left[\% X_{0}\right]$ \\
\hline 1 & \multirow{2}{*}{ SPD } & 3.9 & 14.1 & 80 & 1.14 \\
2 & & 7.6 & 14.1 & 160 & 1.14 \\
3 & & 15.0 & 22.2 & 84 & 1.13 \\
4 & SDD & 23.9 & 29.7 & 176 & 1.26 \\
5 & & 38.0 & 43.1 & 748 & 0.83 \\
6 & SSD & 43.0 & 48.9 & 950 & 0.83 \\
\hline
\end{tabular}

Table 1: Main ITS geometrical parameters.

$p^{+} n$ reverse biased diodes with the pixel size of 50 and $425 \mu \mathrm{m}$ in the bending plane $(r \varphi)$ and beam direction $(z)$ respectively. The nominal bias voltage is $-50 \mathrm{~V}$. The readout is done in the binary mode with the clock frequency of $10 \mathrm{MHz}$. The intrinsic resolution of the SPD module is 12 and $100 \mu \mathrm{m}$ in the $r \varphi$ plane and along $z$ direction respectively.

Silicon Drift Detector (SDD) The SDD consisting of 260 modules occupies the two intermediate layers of the ITS. Each module is composed of a $300 \mu \mathrm{m}$ thick silicon drift sensor and two hybrid boards with the readout chips. The electrical drift field of about $500 \mathrm{~V} / \mathrm{cm}$ parallel to the surface of the sensor is created by the central cathode strip biased to $-1.8 \mathrm{kV}$. This field makes the electrons from ionization drift perpendicularly to the beam axis towards the collection anodes. 512 collection anodes spaced by $294 \mu \mathrm{m}$ are located on two sides of the sensor parallel to the beam axis. In the readout chips the anode signals are digitised by 10-bit ADCs at the sampling frequency of $20 \mathrm{MHz}$. The resulting intrinsic resolution of the SDD is 35 and $25 \mu m$ in the $r \varphi$ plane and along $z$ direction respectively. Moreover, the ADC readout allows to measure the specific energy loss $d E / d x$ of each particle. This information is used for particle identification (PID).

Silicon Strip Detector (SSD) The two outermost layers are equipped with the 1698 modules of the SSD. The SSD module is composed of a $300 \mu \mathrm{m}$ thick double-sided silicon strip sensor and two hybrid boards with the readout chips. The strips on the P-side and the $\mathrm{N}$-side of the sensor are oriented at an angle of 7.5 and $27.5 \mathrm{mrad}$ with respect to the beam axis correspondingly. Strips are $40 \mathrm{~mm}$ long and have $95 \mu \mathrm{m}$ pitch. Signals from the strips are digitised by the 12-bit ADCs. The resulting intrinsic resolution of the SSD is 20 and $830 \mu \mathrm{m}$ in the $r \varphi$ plane and along $z$ direction respectively. Thanks to the ADC readout SSD also provides $d E / d x$ information for particle identification.

The main parameters of the ITS sub-detector modules discussed above are summarized in Table \.

\section{ITS operation and performance during the LHC Run 1}

The ITS was installed in the ALICE experimental cavern in spring 2007. Then, during 20072009 the detector was commissioned and tested with cosmic particles. Finally, in December 2009 the ITS started taking data right from the beginning of the LHC Run 1.

The number of operational modules remained stable during the Run 1 for the SDD and SSD. The SDD had $87 \%$ of the modules taking data. The remaining modules were excluded due to the 


\begin{tabular}{cccc}
\hline & $\begin{array}{c}\text { Cell size } \\
(r \varphi \times z)\left[\mu m^{2}\right]\end{array}$ & $\begin{array}{c}\text { Intrinsic resolution } \\
(r \varphi \times z)\left[\mu m^{2}\right]\end{array}$ & $\begin{array}{c}\text { Active area } \\
{\left[\mathrm{mm}^{2}\right]}\end{array}$ \\
\hline SPD & $50 \times 425$ & $12 \times 100$ & $12.8 \times 69.6$ \\
SDD & $202 \times 294$ & $35 \times 25$ & $72.5 \times 75.3$ \\
SSD & $95 \times 4 \cdot 10^{4}$ & $20 \times 830$ & $73 \times 40$ \\
\hline
\end{tabular}

Table 2: Main parameters of the ITS sub-detector modules.

problems with the high voltage connections or with the Front-End Electronics (FEE) configuration. The SSD had $91 \%$ of the modules active. The other modules were excluded due to excessive noise or problems with the FEE configuration.

The number of the operational modules in the SPD was changing significantly during Run 1. The detector was $100 \%$ operational before its installation in the cavern. At the first powering in the cavern, however, $13 \%$ of the modules had to be switched off because of overheating. The temperature of the modules was raising due to insufficient coolant flow in some sectors of the SPD. Despite several attempts to restore the normal flow the situation was deteriorating with time. In November 2011 only $61 \%$ of the SPD modules remained operational. The analysis of the situation and laboratory tests showed[ם] that the coolant flow was reduced due to clogged filters in the cooling lines. As these filters are installed in the non-accessible place, their replacement would have required partial dismounting of the surrounding ALICE detectors. Therefore this operation was considered not feasible. Several attempts to clean the filters with chemical solutions or with ultrasound were unsuccessful. Finally, it was decided to perforate the filters by drilling. A dedicated procedure had been developed and tested in the laboratory [0]. The first successful drilling was done in February 2012. The filters of the five out of 10 SPD cooling sectors were perforated by April 2012. This allowed recovery of $80 \%$ of the modules reaching the total fraction of the operational modules of $92.5 \%$.

Apart from the SPD cooling problem no major issues were encountered during the Run 1 operations. The ITS was running stably over time. It participated in $96 \%$ of the runs taken by ALICE.

One of main tasks of the ITS is to separate the particles originating from the secondary decay vertices from those coming from the primary collision vertex. This is done by measuring the distance of the closest approach of the particle track to the primary vertex (i.e. the track impact parameter). The track impact parameter resolution in the $r \varphi$-plane as a function of the transverse momentum $p_{T}$ is shown in Fig. $\square$ for three different collision systems. For all of them the impact parameter resolution is $\sim 55 \mu \mathrm{m}$ at $1 \mathrm{GeV} / \mathrm{c}$.

Such a good precision of the ITS allowed the inclusive reconstruction of various rare particles via their hadronic decays. For example, the production of the $\mathrm{D}^{0}, \mathrm{D}^{+}$and $\mathrm{D}^{*+}$ mesons was measured in the central $\mathrm{Pb}-\mathrm{Pb}$ collisions in the transverse momentum range of $2-16 \mathrm{GeV} / \mathrm{c}$. It allowed to calculate the nuclear modification factor $\left(R_{A A}\right)$ [目] and to measure the azimuthal anisotropy [四] of the charmed mesons in $\mathrm{Pb}-\mathrm{Pb}$ collisions.

By measuring the ionizing energy loss $(d E / d x)$ of the particles in the SDD and SSD the ITS contributes to particle identification in ALICE. The ITS is able to identify the low-momentum par- 


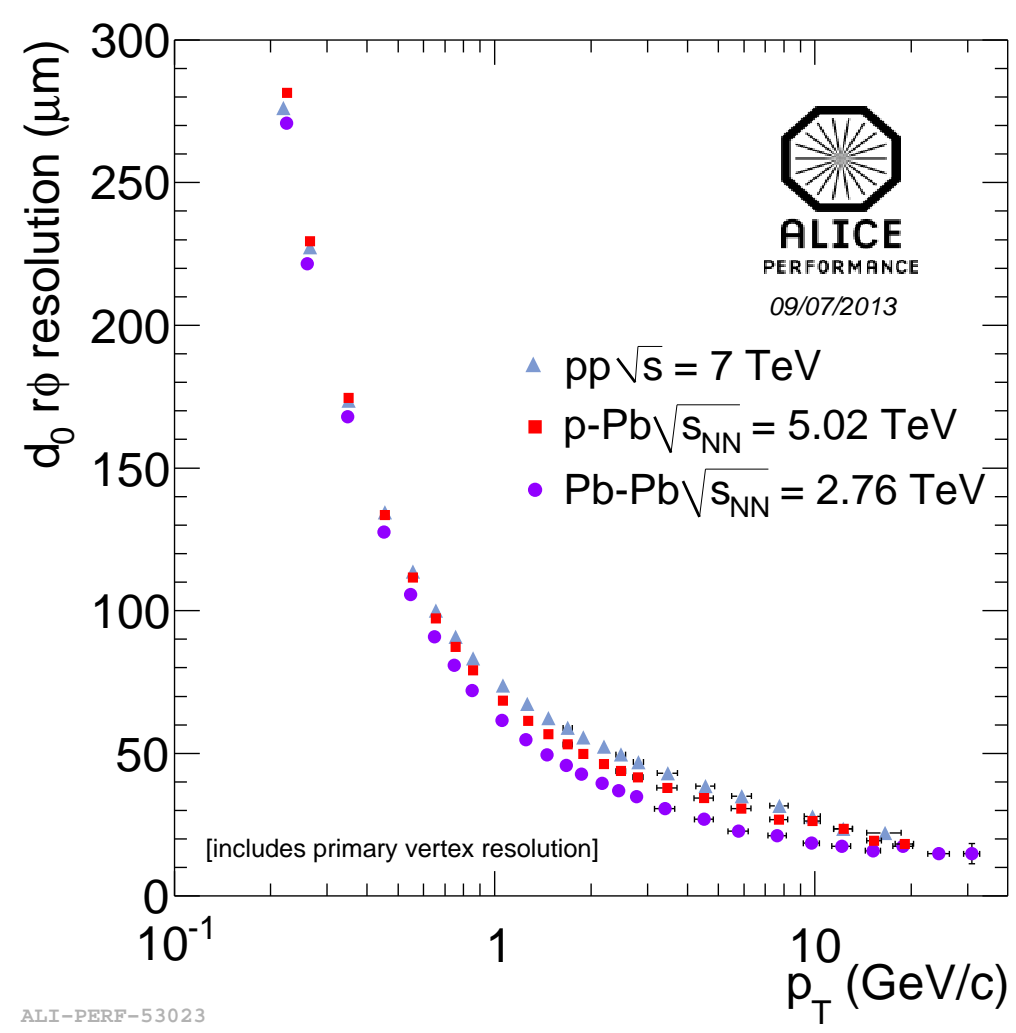

Figure 2: The track impact parameter resolution in the bending plane $(r \varphi)$ as a function of the transverse momentum $p_{T}$ for charged particles.

ticles that can't reach the other more distant detectors. The distribution of the $d E / d x$ for the tracks reconstructed in the ITS is shown in Fig. B]. The distribution features several distinct bands corresponding to different particle species. One can see that K- $\pi$ separation is feasible up to $0.45 \mathrm{GeV} / \mathrm{c}$ and K-p separation up to $1 \mathrm{GeV} / \mathrm{c}$. Such performance allowed to measure the production of $\pi^{ \pm}$, $\mathrm{K}^{ \pm}, \mathrm{p}$ and $\overline{\mathrm{p}}$ in $\mathrm{Pb}-\mathrm{Pb}$ collisions [5]].

More information on the ITS performance can be found in [焑].

\section{ITS consolidation during the first LHC Long Shutdown (LS1)}

Despite the satisfactory performance of the ITS during the LHC Run 1 several detector issues had to be addressed. This was done during the first LHC Long Shutdown (LS1). LS1 started on 14 February 2013 and lasted about 20 month until the end of 2014.

The main task for the SPD was to perforate the filters of the remaining five sectors of the detector. Even if no particular problems were found there, it would allow to prevent possible clogging of the filters in the future. The operation was performed in several sessions. The last two sectors were drilled in January 2014. After this, uniform flow was established in all sectors.

The upgrade of the Detector Data Link (DDL) boards was done for the SDD. These boards connect the detector FEE with the ALICE DAQ system. Previously used boards called CARLOS-rx (Compression And Run Length encOding Subsystem receiver) featuring three Virtex-II FPGAs 


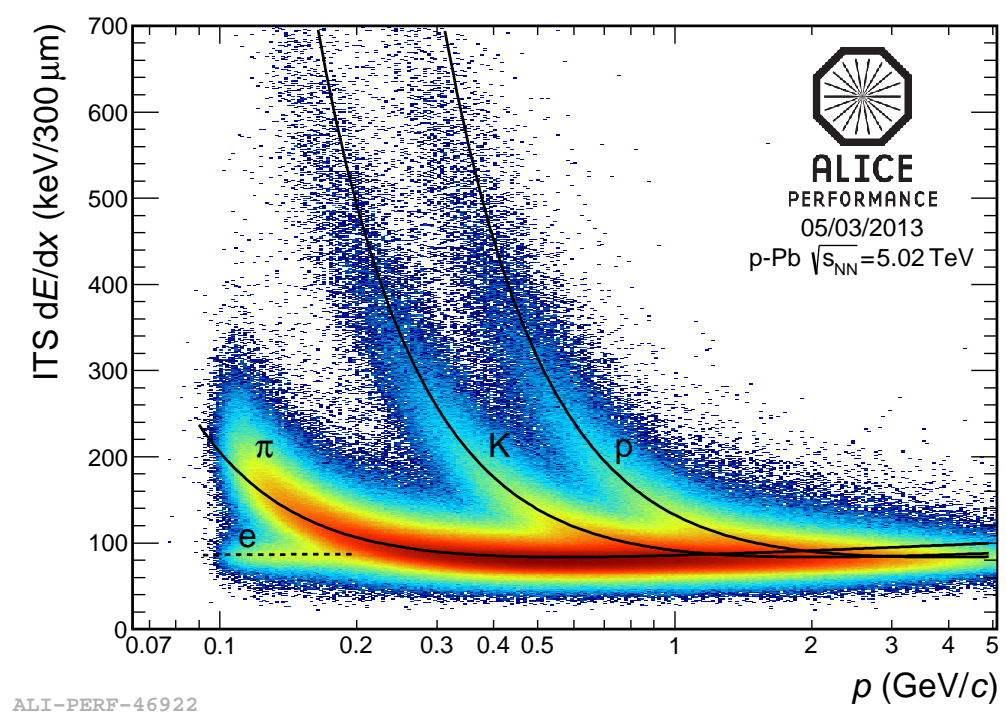

Figure 3: $\mathrm{dE} / \mathrm{dx}$ of charged particles as a function of their momentum for ITS pure standalone tracks measured in $\mathrm{p}-\mathrm{Pb}$ collisions at $5.02 \mathrm{TeV}$.

had limited computing power needed to perform efficient data reduction. The new generation boards called SuperCARLOS-rx have five Virtex-5 FPGAs. They will allow to improve the data compression and increase the flexibility of the system.

The main issue affecting the SSD during the Run 1 was that of Single Event Upsets (SEUs) observed in the SSD Front-End ReadOut Modules (FEROM). These boards are installed in the experimental cavern where they can suffer from the harsh radiation environment. Seven events were observed during the Run 1. It was discovered that they were mostly caused not by the particles produced in the collisions but by the beam induced background from the LHC. This background can get higher during the Run 2 following the increase in LHC luminosity. Several solutions were proposed for this problem. The proposal to shield the affected racks was not considered practical as it would require complicated installations. The adopted solution includes the upgrade of the onboard PROM chips with their radiation hard version and firmware modification allowing for faster recovery of the board.

All the three sub-detectors had their various firmware and software components updated following the ALICE-wide upgrade of the DAQ and detector control systems. Presently the ITS is being re-commissioned for the upcoming LHC Run 2.

\section{Conclusions}

All the components of the ITS performed according to the expectations during the LHC Run 1. Several hardware issues that were observed during operation were properly and timely addressed and solved. The ITS performance contributed to important physics results like measuring production of different particle species in $\mathrm{pp}, \mathrm{Pb}-\mathrm{Pb}$ and $\mathrm{p}-\mathrm{Pb}$ collisions at LHC. After general consolidation during the LHC Long Shutdown 1 the ITS is ready for the LHC Run 2. 


\section{References}

[1] K. Aamodt et al. [ALICE Collaboration], The ALICE experiment at the CERN LHC, JINST 3 (2008) S08002

[2] A. Francescon et al., Performance of the ALICE SPD cooling system, J. Phys. Conf. Ser. 395, 012063 (2012).

[3] B. Abelev et al. [ALICE Collaboration], Suppression of high transverse momentum D mesons in central Pb-Pb collisions at $\sqrt{s_{N N}}=2.76 \mathrm{TeV}$, JHEP 1209 (2012) 112 [arXiv: 1203 .2160]

[4] B. Abelev et al. [ALICE Collaboration], Azimuthal anisotropy of D meson production in $\mathrm{Pb}-\mathrm{Pb}$ collisions at $\sqrt{s_{N N}}=2.76$ TeV, Phys. Rev. C 90 (2014) 034904 [arXiv: 1405.2001 ]

[5] B. Abelev et al. [ALICE Collaboration], Pion, Kaon, and Proton Production in Central Pb-Pb Collisions at $\sqrt{s_{N N}}=2.76$ TeV, Phys. Rev. Lett. 109 (2012) 252301 [arXiv:1208.1974]

[6] B. Abelev et al. [ALICE Collaboration], Performance of the ALICE Experiment at the CERN LHC, Int. J. Mod. Phys. A 29, 1430044 (2014) [arXiv: 1402 . 4476 ] 